\title{
Circadian rhythm of heart rate and physical activity in nurses during day and night shifts
}

\author{
Corinne Nicoletti • Christian Müller · Chiemi Hayashi • \\ Masaru Nakaseko · Itoko Tobita • Thomas Läubli
}

Received: 11 September 2014 / Accepted: 13 January 2015 / Published online: 23 January 2015

(C) Springer-Verlag Berlin Heidelberg 2015

\begin{abstract}
Purpose The study investigates if the circadian rhythm of heart rate is apparent during the working periods of day and night shifts in Swiss and Japanese nurses and if it is influenced by work organization. For a better interpretation of the heart rate, the activity profile over these working periods was monitored.

Methods Heart rate and activity profile of 18 Swiss and 24 Japanese nurses were measured during one day and one night shift. The day and the night shift data of each subject were combined, resulting in an approximately 18-h working period.

Results A significant time effect of the mean hourly value of the heart rate was found in Swiss nurses (change in amplitude $7.1 \mathrm{bpm}$ ) as well as in Japanese nurses (11.8 bpm). These effects could be modeled with cosine curves for the Swiss and Japanese subjects. For the activity level significant time effects, similar to the ones in heart
\end{abstract}

Communicated by Dick F. Stegeman.

C. Nicoletti $(\bowtie) \cdot$ T. Läubli

Department of Health Sciences and Technology, ETH Zurich, Sonneggstrasse 3, 8092 Zurich, Switzerland

e-mail: corinne.nicoletti@hest.ethz.ch

C. Müller

Occupational Health Management, SBB AG, Swiss Federal

Railways, Hochschulstrasse 6, 3000 Bern, Switzerland

C. Hayashi · M. Nakaseko $\cdot$ I. Tobita

Occupational Ergonomic Unit, Management in Health Care

Sciences, Graduate School of Health Sciences, Jikei Institute,

1-2-8 Miyahara, Yodogawaku, Osaka 532-0003, Japan

M. Nakaseko · T. Läubli

Holistics Prosthetics Research Center, Kyoto Institute

of Technology, Matsugasaki, Sakyo-ku, Kyoto 606-8585, Japan rate, were found in Swiss nurses (87\% of SD) but not in Japanese nurses (26\% of SD).

Conclusions We found a significant time effect in heart rate similar to the known circadian rhythm under normal sleep-wake conditions while working in the studied shift work schedules. In the Japanese nurses, studied heart rate followed a circadian rhythm independently of the level of physical activity. Therefore, an activity profile following the circadian rhythm, especially a reduced workload from 2 to 4 a.m., is proposed. The proposed activity profile could be reached with an adapted work organization.

Keywords Shift work - Circadian rhythm - Heart rate . Nurse · Activity
Abbreviations
ECG Electrocardiography
EMG Electromyography

\section{Introduction}

All over the world, workers are involved in night work. In Europe and the USA, 15-20\% of the working population is involved in shift work including night shifts (Straif et al. 2007). With an amount of more than $30 \%$, the health care sector is one of the sectors in which shift work including night shifts is most prevalent. Shift work, especially if it includes night work, is known to be disruptive for the circadian rhythm (Bonde et al. 2012; Bracci et al. 2014; Straif et al. 2007).

On one side, the circadian rhythm is given by an internal pacemaker called master circadian system and located in the suprachiasmatic nucleus. On the other side, the circadian rhythm is strongly influenced by environmental 
clues, like the light-dark cycle (Ruger and Scheer 2009). This results in a complex system of many different regulation mechanisms (Bollinger and Schibler 2014). If the internal rhythm, given by the master circadian system and the light-dark cycle is shifted, a circadian misalignment occurs (Bonde et al. 2012; Ruger and Scheer 2009). This desynchronization leads to a disturbance in melatonin secretion. During a regular night, melatonin is secreted in high levels. Exposure to light during this biological sleeping period, leads to a misalignment of the circadian rhythm and thereby to a desynchronization of the melatonin secretion (Bonde et al. 2012).

Different health problems are associated with shift work. The most prominent one is the increased prevalence of different cancer types (Parent et al. 2012; Schernhammer et al. 2003), especially of breast cancer (Bonde et al. 2012; Leonardi et al. 2012). Estryn-Behar et al. (2008) found an association of shift work including night shifts and burnout. Läubli and Müller (2009) found an correlation of shift work and musculoskeletal disorders. Associations can also be found with sleep disturbances (Flo et al. 2012; Guo et al. 2013; Oyane et al. 2013), chronic fatigue (Oyane et al. 2013), changes in behavior pattern, depression, digestive disorders and cardiovascular diseases (Costa 1996; Vyas et al. 2012). Bonde et al. (2012) state in their review that "disruption of the circadian rhythm is assumed to be a main pathway from shift work to disease". To reduce the disruption and therefore also the health problems, several authors propose rapidly rotating shift systems (Bonde et al. 2012; Knauth et al. 1979).

Heart rate is one of the physiological parameters underlying a circadian rhythm (Ruger and Scheer 2009). Data of the circadian rhythm of heart rate in healthy subjects under normal sleep-wake conditions are rarely reported. The reported data show a peak about noon to afternoon and a minimum about 3-6 a.m. (Clarke et al. 1976; Huikuri et al. 1990). Beside the circadian rhythm heart rate is also influenced by physical (Grandjean 1991) and psychological loads (Fisher and Newman 2013). Several studies measured heart rate during shift work and also during leisure time in shift workers (Colquhoun 1988; Goto et al. 1994; Ito et al. 2001), but working nights were rarely compared with working days. These kind of measurements are important to understand the interaction between activities at work and HR variability. However, to the best of our knowledge none of the above-mentioned studies investigated the circadian rhythm and controlled heart rate simultaneously for time of the day and workload.

With this study, we have in mind the claim of Bonde et al. (2012) who declared that "it is important to identify and implement shift systems that minimize circadian disruption". As a first step towards a shift system that reduces circadian disruption, we intend to clarify in two work situations if over an approximately 18-h working period, consisting of a combined day and night shift, the circadian rhythm of heart rate is apparent. We also aim to compare the changes of heart rate and of activity profiles during the different hours of the working period.

\section{Materials and methods}

\section{Ethics statement}

The ethical committee of the canton of Zurich (Switzerland) approved the Swiss study and all Swiss subjects gave their written informed consent. In the Japanese study, the ethical committee of the Japan Health and Welfare Organization (580-1 Horikawacho, Saiwaiku, Kawasakishi, Kanagawa 212-0003, Japan) approved this study. All Japanese subjects gave their written informed consent. Subjects were instructed according to the Helsinki Declaration, participated voluntarily and were free to discontinue their participation at any time without explanation.

\section{Subjects}

18 nurses participated in the Swiss and 24 nurses in the Japanese study (Table 1). They were all female and had an education equal to a registered nurse (Table 1). All subjects worked at least for 1 year on the job. Swiss subjects worked either part-time ( $\geq 80 \%$ ) or full-time and Japanese subjects worked full-time. Subjects fulfilling the following criteria were excluded: clinical findings of MSDs, intake of muscle relaxants and subjects with skin disease. In the Swiss dataset subjects with cardiovascular, psychological or neurological diseases, or subjects under medication were excluded. In the Japanese datasets, subjects with shoulder and neck pain due to injury or systemic disease, subjects using tranquilizing medication and subjects with a BMI $>30$ were excluded. The Swiss nurses were selected based on their willingness to participate and if their regular work schedule included day and night shifts. In all subjects the unit agreed with the proposed study. In Japanese nurses agreement was obtained from a participating unit and afterwards the subjects were selected based on their willingness to participate and if they regularly worked day and night shifts. The working hours of the measured shifts are shown in Table 1. Additionally, the shift systems differed between the two countries: in Japan the night shift started $7 \mathrm{~h}$ and $30 \mathrm{~min}$ after the end of the previous day shift. In Swiss nurses the night shift started on the next day, more than $24 \mathrm{~h}$ after the ending of the last day shift.

\section{Apparatus}

Heart rate of the Swiss subjects was collected with a two electrodes electrocardiogram (ECG; sampling rate 
Table 1 Specifications of Swiss and Japanese subjects

\begin{tabular}{lll}
\hline Data set & Swiss subjects & Japanese subjects \\
\hline Data collection & $6.2011-11.2011$ & $10.2010-2.2011,11.2011-2.2012$ \\
$N$ & 18 & 24 \\
Age & $31.3 \pm 8.2$ & $31.0 \pm 8.2$ \\
Ward & Orthopedic ward $(n=9)$ & Cardiology ward $(n=6)$ \\
& Intensive care $(n=6)$ & Orthopedic ward $(n=9)$ \\
& Emergency department $(n=2)$ & Circulation and lung $(n=5)$ \\
& Post-anesthesia care unit $(n=1)$ & Surgery $(n=4)$ \\
Day shift & $7.00-16.00$ & $08.00-16.30(n=6) / 08.30-17.00(n=18)$ \\
Night shift & $21.30-7.00(n=9) / 23.00-7.30(n=6) / 22.00-6.00$ & $00.00-8.30(n=6) / 00.30-09.00(n=18)$ \\
& $(n=2) / 20.00-6.30(n=1)$ & \\
Order of the shifts & Randomly; only one shift per day & Day shift and later on the same day night shift \\
\hline
\end{tabular}

1,000 Hz) using the electromyography (EMG) recording device PS11-UD (THUMEDI GmbH \& Co. KG, ThumJahnsbach, Germany). The ECG was directly processed with the device (moving average mean over $7 \mathrm{R}-\mathrm{R}$ intervals), thus heart rate was obtained. The electrodes used were pre-gelled $\mathrm{Ag} / \mathrm{AgCl}$ electrodes (Kendall Arbo, England). In the Japanese dataset, heart rate was measured with a heart rate watch (Polar CS600, Polar, Finland) with a sampling rate of $2,400 \mathrm{~Hz}$.

For the Swiss subjects, the acceleration of the dominant upper arm was also measured with the PS11-UD. A onedimensional accelerometer (sampling rate $8 \mathrm{~Hz}$ ) was fixed on the upper arm. Activity of the Japanese subjects was detected using the ViM sports memory (MicroStone, Japan) with a sampling rate of $24 \mathrm{~Hz}$. The ViM sports memory was fixed on the dominant upper arm. Using a built-in algorithm, it classified ten different types of movements based on registrations by a three-dimensional accelerometer and a gyroscope. The ViM sports memory analyzed consecutive 3-min intervals and calculated the temporal distribution of these ten different movement types within the 3-min intervals.

\section{Procedure}

Each subject was measured during a day and a night shift on the same ward (Table 1; Swiss subjects were measured during 2 day shifts, but for this analysis only data of the first day shift were used). For the Swiss subjects, the order of the measured shifts was random. In Japanese subjects, the day shift was always measured directly before the night shift. The night shift measured in Swiss subjects was the second or in one case the third in a row. In Japanese subjects, the measured night shift was the first in a row.

The measurement device was applied before work started. In Swiss subjects, one electrode of the ECG was placed on the left side of the chest wall below the breast and the other one below the clavicle. The accelerometer was worn at the upper arm and was fixed with tape. The measuring device was worn around the waist and the cables were fixed on the skin for reducing movement artifacts. For the Japanese data, the belt of the heart monitoring watch was worn around the chest and the watch around the wrist. The ViM sports memory was worn on the dominant upper arm.

\section{Analysis}

All data were processed with Matlab R2011b. For the heart rate data of Swiss subjects, the PS11-UD used an internal filtering procedure. This procedure removed values smaller than $30 \mathrm{bpm}$, bigger than $200 \mathrm{bpm}$ and if the standard deviation of 7 values was bigger than $40 \mathrm{bpm}$. Heart rate of Japanese subjects was analyzed with the help of peak detection and visual inspection and artifacts were removed. The heart rate data of every subject was split in 3-min intervals. For every 3-min interval the median was calculated. The data from the day and the night shift of every subject were combined, covering approximately $18 \mathrm{~h}$.

For building the line with the median value (thick line) in Fig. 1a, b, for every 3-min interval, the median of heart rate over all Swiss (Fig. 1a) or Japanese (Fig. 1b) subjects was calculated. Of these median values, a moving average mean over 5 values, or $15 \mathrm{~min}$, was calculated. These values were used in Fig. 1 as median (thick line). To describe the minimum and the maximum heart rate values (thin lines) in Fig. 1, for every hour and for every subject, the smallest and the biggest value of the 23-min intervals was searched and written in a new variable with its real time. These minimum and maximum values were also averaged over all Swiss or Japanese subjects using the median. A moving average over five values was calculated. These values are used in Fig. 1a, b as minimum and maximum values (thin lines). As the time intervals of the minimum and 

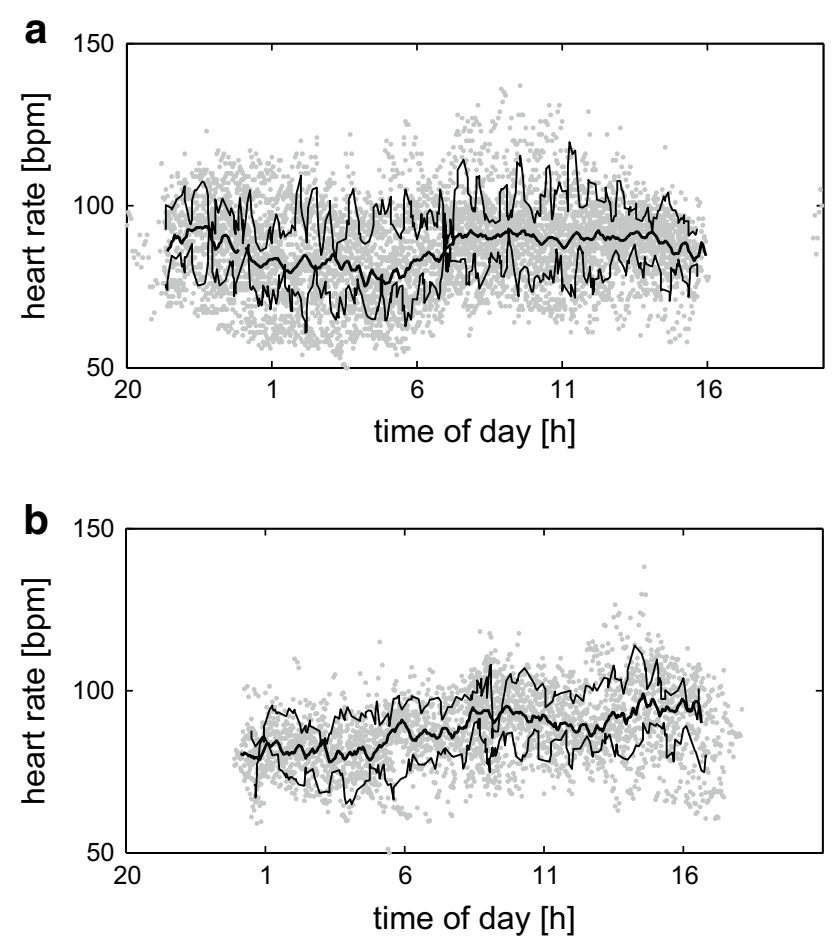

Fig. 1 3-min-averaged heart rate values of the combined day and night shift of a Swiss and b Japanese subjects. The 3-min-averaged heart rate values are shown as closed dots. The thick line shows the moving average mean values of the subjects and the thin lines show the moving average minimum and maximum values of the subjects

maximum values were not constant within the single hours, the distribution of these values is not constant. For statistical purpose, the median of heart rate values over $1 \mathrm{~h}$ was calculated for every subject (using the 3-min intervals). The first interval started at 0.30 a.m.

For arm acceleration of Swiss subjects, 3-min intervals were generated using the median. In Japanese data, the built-in algorithm of the measuring device calculated for every 3-min interval the percentages of the following movement types: "normal walking", "fast walking", "very fast walking", "light running", "fast running", "light sport" and "intensive sport", "desk work", "standing work" and "slow walking". For our analysis, "normal walking", "fast walking", "very fast walking", "light running", "fast running", "light sport" and "intensive sport" were classified as "active" and "desk work", "standing work" and "slow walking" as "not active". For every 3-min interval, the percentage of "active" tasks was calculated. From the resulting 3-min interval values of both countries, the mean of all Swiss or Japanese subjects was obtained. From these values, the moving average over 5 values was calculated. Afterwards, these values were z-transformed (i.e., the mean was deducted from every value and the resulting value was divided by the standard deviation) and plotted in Fig. 3. For statistical analysis of activity data, the mean over $1 \mathrm{~h}$ was calculated for every subject. The first 1-h interval started at 0:30 a.m.

Two different measuring devices were used to measure heart rate and their reliability was checked. A test person wore both devices for $2 \mathrm{~h}$, simulating typical activities of a nurse. A correlation of $R=0.85$ was reached. An $R=0.9$ was reached after exclusion of rare artifacts that were caused because the devices touched each others.

\section{Statistics}

General linear model analysis was used for statistical analyses of heart rate and activity (SAS 9.2). The ward the nurses worked on was used as a class variable. The Greenhouse-Geisser Epsilon was chosen for the evaluation of significance (Bortz and Schuster 2010). A cosine curve was fitted on the 1-24 h for heart rate and activity of Swiss and Japanese subjects. The amplitude, the phase shift and the displacement of the cosine curve were fitted. The period was set to 0.25 , because we fitted the model to a $24 \mathrm{~h}$ rhythm.

\section{Results}

Combined day and night shifts were used for heart rate analysis (separately for the 18 Swiss and 11 Japanese subjects) and for the analysis of activity (separately for the 18 Swiss and 23 Japanese subjects). In Swiss data, $5.9 \pm 11.9 \%$ of heart rate data per subject were excluded and in Japanese data it was $1.8 \pm 2.8 \%$.

\section{Heart rate}

Figure 1 shows the heart rate over the combined day and night shift period for (a) Swiss and (b) Japanese nurses. The dots represent the single 3-min values of all subjects. The thick line shows the moving average mean value of the 3 -min intervals of the subjects. The two thinner lines indicate the moving average of minimum and maximum values of every hour of the subjects. A highly significant time effect of the hourly median values of heart rate was found for the Swiss $(F=6.57, p<0.001$ with Greenhouse-Geisser Epsilon) as well as for the Japanese nurses $(F=7.65$, $p<0.001$ with Greenhouse-Geisser Epsilon).

Additionally, a cosine curve was fitted on the heart rate data of the Swiss $\operatorname{hr}(t)=-3.5 \times \cos (0.25 \times t+12.2)+$ $86.1\left(R^{2}=0.05, F=8.03\right)$ and of the Japanese subjects $\operatorname{hr}(t)=-5.9 \times \cos (0.25 \times t+12.4)+86.4\left(R^{2}=0.18\right.$, $F=19.7)$. 

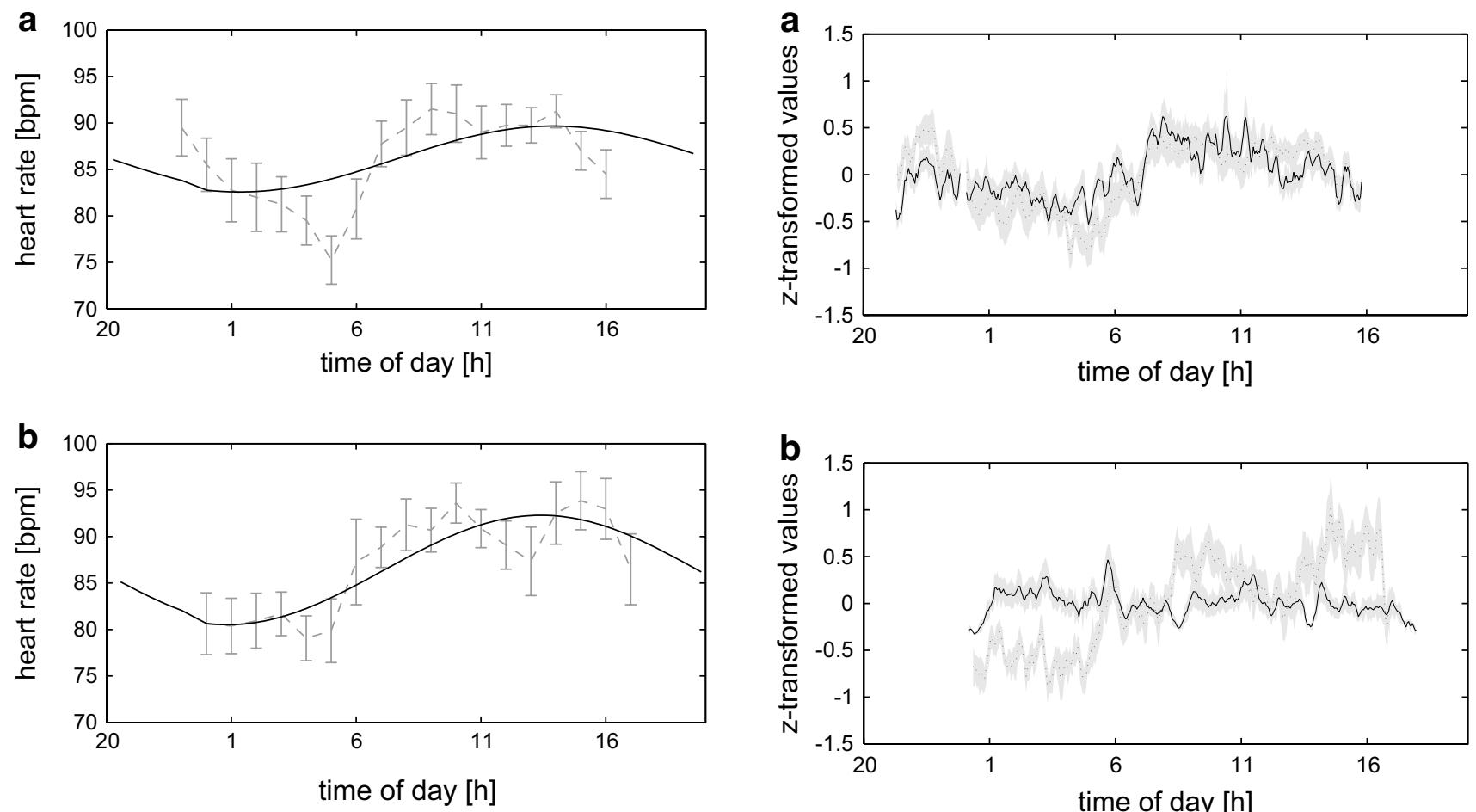

Fig. 2 Heart rate and the fitted cosine curve of a Swiss and b Japanese subjects. Hourly mean values of heart rate of the subjects with the standard error are shown as a grey dashed line and the fitted cosine function as a black solid line

The 1-h mean values of the heart rate of the subjects and the fitted cosine curves are shown in Fig. 2a for the Swiss and Fig. $2 \mathrm{~b}$ for the Japanese nurses.

Both groups showed a drop in heart rate in the early morning. The subjects showed the minimum around midnight to 3 a.m. and a wide maximum between 12 a.m. and 3 p.m.

\section{Activity}

No difference in activity between the different wards within a country was found [Japanese part $p=0.9$ with one-way anova, Swiss part $p=0.4$ (for trapezius muscle activity and details in Swiss nurses see Nicoletti et al. (2013))] thus it is justified to jointly analyze the nurses from different wards within the countries. Analyzing the activity pattern of the Japanese nurses no significant differences between the different hours of the day were found $(F=1.04, p=0.36$ with Greenhouse-Geisser Epsilon). In the data of Swiss nurses, significant time-dependent differences of activity were found $(F=5.22, p<0.001$ with Greenhouse-Geisser Epsilon).

Figure 3 shows the z-transformed values of activity and heart rate of Swiss and Japanese data. In Swiss data, the curves of heart rate and activity were similar. In Japanese

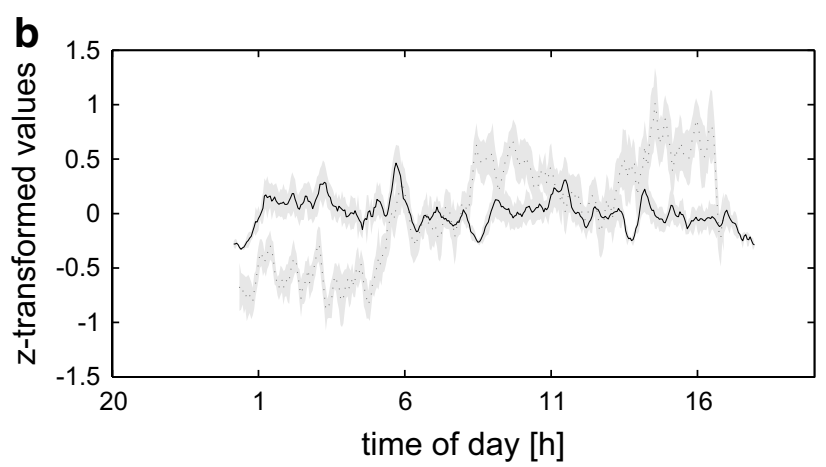

Fig. 3 Z-transformed values of heart rate and activity of a Swiss and $\mathbf{b}$ Japanese nurses. Heart rate is shown as a grey dashed line and activity as a black solid line. For both parameters the standard error is shown as a grey area

data, as shown by the statistical analysis, activity presented no time-dependent effect.

We additionally fitted a cosine curve on the activity of Swiss subjects: activity $(t)=-0.12 \times \cos$ $(0.25 \times t+13.0)+0.61\left(R^{2}=0.10, F=18.45\right.$, change in amplitude $87 \%$ of the standard deviation)

For the Japanese subjects fitting a cosine curve with a period of a full day did not give a significant result $\left(R^{2}=0.01, F=1.81\right.$, change in amplitude $26 \%$ of the standard deviation). As already seen above, the activity of the Japanese nurses did not follow a 24-h rhythm $(F=1.81)$. In the Swiss nurses we found a significant $24-\mathrm{h}$ rhythm $(F=18.45)$ with a similar phase shift as in the heart rate (with a phase of 13 for the activity and 12.2 for the heart rate).

\section{Discussion}

In the early morning a significant drop in heart rate was observed in the Japanese as well as in the Swiss nurses that can be explained by the circadian rhythm. In the Japanese nurses, the circadian drop was present in spite of a continuously high activity level. 
Swiss and Japanese nurses showed a significant timedependent variation in heart rate during work. This timedependent variation was similar to the common circadian rhythm under normal sleep-wake conditions (Clarke et al. 1976; Huikuri et al. 1990). The amplitude of the rhythm is smaller in the Swiss than in the Japanese nurses. This may be due to the work schedule, as the measured night shift was the second one in Swiss, but the first one in Japanese nurses. Considering the fitted cosine curve, the Swiss as well as the Japanese nurses showed a minimum in heart rate from midnight till $3 \mathrm{a} . \mathrm{m}$. The data additionally showed a local minimum at 5 a.m. in the Swiss nurses and at 4 a.m. in the Japanese ones. Especially in the Swiss nurses, where heart rate was at least $5 \mathrm{bpm}$ lower than in every other point in time, it is probable that at $5 \mathrm{a} . \mathrm{m}$. the nurses had a period with less work. Especially because at 5 a.m. the activity level of the Swiss nurses also was quite low. In the Japanese nurses, the drop at 4 a.m. is smaller and may also be due to chance.

Goto et al. (1994) found in nurses a significantly lower heart rate during night shift than during day shift. Ito et al. (2001) concluded in their study that only a small contribution of the internal clock to the circadian rhythm was apparent, but a large contribution of activity. Ito's study, used heart rate data of ten Japanese nurses, but did not include the work activities of the nurses in the analysis. They only included a division in sleeping, working or leisure time. In Ito et al. (2001), heart rate had the smallest values at 4 a.m. and the highest at 2 p.m. with a difference of approximately $25 \mathrm{bpm}$ that is a bigger difference than in our study. Furthermore, heart rate variation was similar to the typical circadian variation. Colquhoun (1988) examined 8-h sessions of sedentary shift work and with an increasing number of consecutive night shifts he found an adjustment of the heart rate to the timing of the shift and an elevation in heart rate after big meals. So a rhythm of heart rate, similar to the circadian rhythm seems to be maintained during shift work but may be disturbed by special duties or with increasing number of night shifts.

In Japanese nurses, the averaged heart rate seems to be independent of the activity. The activity level during the night shift was high, maybe even slightly higher than during day shift. Despite the high activity, heart rate was rather low. As a methodological point for further research, we state that the activity level during night shift could be underestimated only using heart rate. Therefore, additional parameters of activity, like accelerations or changes in positions should be recorded.

Bearing in mind the cancer problematic discussed in the introduction, the demands on the activity level during the night shifts should be reconsidered. The review of Bonde et al. (2012) mentioned various factors that causes circadian disruption and therefore could result in an elevated risk for breast cancer in night shift workers. The review mentioned the type of shift system, behavioral and lifestyle factors and environmental factors. The light exposure during night shifts seems clearly to be the most important factor, as it regulates the secretion of melatonin. Considering our results, we propose to see the activity level during night shifts as a possible additional influencing factor. When the activity level during night is as high as during day shift, but heart rate could not rise, additional disturbances of the circadian system are possible. Indeed, some evidence can be found that in long-term night work or if travelling over time zones, a high activity level during the biological night force the change of circadian rhythm (Atkinson et al. 2007). Therefore, we propose to adapt the workload during shift work to the circadian rhythm. Activity during night, especially from 2 to 4 a.m. should be reduced. Using more staff could be one possibility to reach this. In Swiss nurses, a period with less work seem to exist at about 5 a.m. This raises the question if it would be possible to shift this period to the time with the minimum of the circadian rhythm, which means from 2 to 4 a.m. Further research in this field is strongly needed.

Also other reasons support the adaption of workload to the circadian rhythm. Santhi et al. (2007) showed a decline in cognitive processes during the night. Riedel et al. (2011) found between 2 and 4 a.m. a critical period with a significantly increased activity adjusted risk to experience a work-related accident. Thus, between 2 and 4 a.m. work duties should be minimized as much as possible to reduce work accidents.

It seems that the organization of shift work can influence the amount of circadian disruption and therefore it should be possible to reduce the disruption of the circadian systems be means of work organization. Based on the results it can be concluded that a high amount of activity during night shifts could augment the circadian disruption. Further studies are needed to clarify this and investigate which additionally factors of the work organization could have an influence on the circadian disruption. A often studied method to reduce workload during night shifts is napping. A review (Ruggiero and Redeker 2014) showed that napping can reduce sleepiness and can improve the sleep-related performance. But the review also showed that naps can lead to sleep inertia. Furthermore, Edwards et al. (2013) showed that nurse managers perceive some conflicts related to napping and that $70 \%$ of the interviewed managers see drawbacks of the napping. Additionally, opportunities for napping do not exist in every hospital. Therefore, another solution to reduce the load and the misalignment due to night shifts is strongly needed.

We are aware, that this is only a small study containing some limitations. The study design was not meant to be a statistical comparison between the effect of different 
shift schedules, but was meant to shed light on the interplay between heart rate and physical activity during night work in two examples. Both data sets were independently analyzed and are displayed in a similar way. Based on a close cooperation between the research teams of the two countries that included reciprocal site-visits we are sure, that we could avoid large differences in the selection of nurses and in the interpretation of the measures taken. Using different measuring devices complicates comparisons of absolute levels of the activity and eventually heart rate, but as generally known does not hinder the analysis of the course within single subjects or here within the Swiss and Japanese nurses. Of course results from two culturally different countries are reported. This seems brave but literature reviews also do not pay great attention to the fact that studies are compiled from many countries. Additionally, we did not record the exact sleep schedule of the participants, the menstrual cycle or the menopausal status, the consumption of tea, coffee or tobacco and the illumination levels. Further research controlling all these parameters is strongly needed. However, we obtained clear and statistically highly significant results and we are convinced that they objectively describe some aspects of the physiological workload in Swiss and Japanese nurses. The study highlights some unsolved issues on the potential interactions between activity levels and heart rate during night work that should be considered in the organization of shift work.

\section{Conclusion}

We conclude that a significant time-dependent variation in heart rate, similar to the known circadian rhythm under normal sleep-wake conditions, can be retained in nurses working night and shift work. In the example of the Japanese nurses heart rate followed a circadian rhythm independently of the level of physical activities and adaptation to the workload seems to be disturbed. Therefore, an activity profile according to the circadian rhythm, which better corresponds to the physiological needs, is proposed. Especially during the critical period from 2 to 4 a.m. work demands should be eased, taking into account the known increased risk of work accidents during this time, and to counteract against disturbances of the circadian rhythm. The organization of the shift work seems to have an influence on the disruption of the circadian system and has to be further examined.

Acknowledgments We thank the Swiss Federal Institute of Technology (ETH) Zurich, the Kyoto Technical Institute and the Graduate School of Health Sciences, Jikei Institute (Osaka, Japan) for their financial support.
Conflict of interest The authors declare that they have no conflict of interest.

\section{References}

Atkinson G, Edwards B, Reilly T, Waterhouse J (2007) Exercise as a synchroniser of human circadian rhythms: an update and discussion of the methodological problems. Eur J Appl Physiol 99:331-341

Bollinger T, Schibler U (2014) Circadian rhythms-from genes to physiology and disease. Swiss Med Wkly 144:w13984

Bonde JP, Hansen J, Kolstad HA, Mikkelsen S, Olsen JH, Blask DE, Harma M, Kjuus H, de Koning HJ, Olsen J, Moller M, Schernhammer ES, Stevens RG, Akerstedt T (2012) Work at night and breast cancer-report on evidence-based options for preventive actions. Scand J Work Environ Health 38:380-390

Bortz J, Schuster C (2010) Statistik für Human- und Sozialwissenschaftler, 7th edn. Springer, Heidelberg

Bracci M, Manzella N, Copertaro A, Staffolani S, Strafella E, Barbaresi M, Copertaro B, Rapisarda V, Valentino M, Santarelli L (2014) Rotating-shift nurses after a day off: peripheral clock gene expression, urinary melatonin, and serum $17-\beta$-estradiol levels. Scand J Work Environ Health 40(3):295-304

Clarke JM, Hamer J, Shelton JR, Taylor S, Venning GR (1976) The rhythm of the normal human heart. Lancet 1:508-512

Colquhoun WP (1988) Heart-rate patterns in sedentary shift workinfluence of circadian-rhythm, meals and personality. Int Arch Occup Environ Health 60:273-278

Costa G (1996) The impact of shift and night work on health. Appl Ergon 27:9-16

Edwards MP, McMillan DE, Fallis WM (2013) Napping during breaks on night shift: critical care nurse managers perceptions. Dynamics (Pembroke, Ont) 24:30-35

Estryn-Behar M, van der Heijden B, Camerino D, Fry C, Le Nezet O, Conway PM, Hasselhorn HM (2008) Violence risks in nursing-results from the European next study. Occup Med Oxf 58:107-114

Fisher AJ, Newman MG (2013) Heart rate and autonomic response to stress after experimental induction of worry versus relaxation in healthy, high-worry, and generalized anxiety disorder individuals. Biol Psychol 93:65-74

Flo E, Pallesen S, Mageroy N, Moen BE, Gronli J, Nordhus IH, Bjorvatn B (2012) Shift work disorder in nurses-assessment, prevalence and related health problems. PLoS One 7:e33981

Goto T, Yokoyama K, Araki T, Miura T, Saitoh H, Saitoh M, Satoh S (1994) Identical blood-pressure levels and slower heart-rates among nurses during night work and day work. J Hum Hypertens 8:11-14

Grandjean E (1991) Physiologische Arbeitsgestaltung. Leitfaden der Ergonomie. Ott Verlag, Thun

Guo Y, Liu Y, Huang X, Rong Y, He M, Wang Y, Yuan J, Wu T, Chen W (2013) The effects of shift work on sleeping quality, hypertension and diabetes in retired workers. PLoS One 8:e71107

Huikuri HV, Kessler KM, Terracall E, Castellanos A, Linnaluoto MK, Myerburg RJ (1990) Reproducibility and circadian rhythm of heart rate variability in healthy subjects. Am J Cardiol 65:391-393

Ito H, Nozaki M, Maruyama T, Kaji Y, Tsuda Y (2001) Shift work modifies the circadian patterns of heart rate variability in nurses. Int J Cardiol 79:231-236

Knauth P, Rohmert W, Rutenfranz J (1979) Systematic selection of shift plans for continuous production with the aid of work-physiological criteria. Appl Ergon 10:9-15 
Läubli T, Müller C (2009) Arbeitsbedingungen und Erkrankungen des Bewegungsapparates-Geschätzte Fallzahlen und volkswirtschaftliche Kosten für die Schweiz. http://www.news.admin. ch/NSBSubscriber/message/attachments/16739.pdf. Accessed 6 sept 2012

Leonardi GC, Rapisarda V, Marconi A, Scalisi A, Catalano F, Proietti L, Travali S, Libra M, Fenga C (2012) Correlation of the risk of breast cancer and disruption of the circadian rhythm (review). Oncol Rep 28:418-428

Nicoletti C, Spengler CM, Laubli T (2013) Physical workload, trapezius muscle activity, and neck pain in nurses night and day shifts: a physiological evaluation. Appl Ergon 45:741-746

Oyane NM, Pallesen S, Moen BE, Akerstedt T, Bjorvatn B (2013) Associations between night work and anxiety, depression, insomnia, sleepiness and fatigue in a sample of Norwegian nurses. PLoS One 8:e70228

Parent ME, El-Zein M, Rousseau MC, Pintos J, Siemiatycki J (2012) Night work and the risk of cancer among men. Am J Epidemiol 176:751-759

Riedel M, Berrez S, Pelisse D, Brousse E, Forget C, Marlot M, Smolensky MH, Touitou Y, Reinberg A (2011) 24-hour pattern of work-related injury risk of French firemen: nocturnal peak time. Chronobiol Int 28:697-705
Ruger M, Scheer F (2009) Effects of circadian disruption on the cardiometabolic system. Rev Endocr Metab Disord 10:245-260

Ruggiero JS, Redeker NS (2014) Effects of napping on sleepiness and sleep-related performance deficits in night-shift workers: a systematic review. Biol Res Nurs 16:134-142

Santhi N, Horowitz TS, Duffy JF, Czeisler CA (2007) Acute sleep deprivation and circadian misalignment associated with transition onto the first night of work impairs visual selective attention. PLoS One 2:e1233

Schernhammer ES, Laden F, Speizer FE, Willett WC, Hunter DJ, Kawachi I, Fuchs CS, Colditz GA (2003) Night-shift work and risk of colorectal cancer in the nurses health study. J Natl Cancer Inst 95:825-828

Straif K, Baan R, Grosse Y, Secretan B, El Ghissassi F, Bouvard V, Altieri A, Benbrahim-Tallaa L, Cogliano V, Monograph WHOIARC (2007) Carcinogenicity of shift-work, painting, and firefighting. Lancet Oncol 8:1065-1066

Vyas MV, Garg AX, Iansavichus AV, Costella J, Donner A, Laugsand LE, Janszky I, Mrkobrada M, Parraga G, Hackam DG (2012) Shift work and vascular events: systematic review and meta-analysis. Br Med J 345:e4800 